\title{
A prediction model for mining subsidence in loess-covered mountainous areas of western China
}

\author{
Fuquan Tang ${ }^{1,2}$, Jiaxin $\mathrm{Lu}^{1}$ and Pengfei $\mathrm{Li}^{1, *}$ \\ ${ }^{1}$ College of Geomatics, Xi'an University of Science and Technology, Xi'an, 710054, China \\ ${ }^{2}$ Key Laboratory of Coal Resources Exploration and Comprehensive Utilization, Ministry of Land and Resources, Xi'an, China
}

\begin{abstract}
Land subsidence in the loess-covered mountainous area is a complex process that contemporary models could not accurately simulate. We assumed that flatground mining subsidence was the result of joint action of bedrock mining subsidence under equivalent load of the loess layer and the spread of bedrock surface subsidence to land surface via thick loess layers. Quantitative relationships between equivalent load of the loess layer and equivalent exploitation width, depth, and bedrock subsidence were examined. A double-medium model of flat-ground mining subsidence based on stochastic medium theory was developed to simulate the interactions between loess layers and bedrock. Another model was established to describe the slip deformation associated with loess in hill side mining. The two models were integrated to account for mining subsidence on flat ground and hillside. The integrated model was demonstrated to be robust in land subsidence deformation prediction for loess-covered mountainous area based on field measurements from a mining area in western China.
\end{abstract}

Keywords: Deformation prediction, loess layer, mining area of western China, mining subsidence, hillside slip.

IN the past several decades, a number of studies have focused on using various methods/models to estimate the ground subsidence and deformation caused by underground coal mining. They include the geometric theory prediction method ${ }^{1}$, profile function prediction method ${ }^{2}$, mechanical model prediction method $^{3}$, and numerical simulation prediction method ${ }^{4}$. China is one of the major coal mining countries in the world, where coal mining subsidence is commonly noticeable $\mathrm{e}^{5-7}$. Since the 1950 s, some efforts have been made to develop mining subsidence prediction models suitable for geological and mining conditions in China. Many models were thus developed including the probability integral method ${ }^{5}$, negative exponential function method, 'Weibull distribution' $\operatorname{method}^{8}$, and elastoplastic mechanics prediction method $^{9}$. Among them, the probability integration method, based on the random medium theory ${ }^{10}$ esta-

\footnotetext{
*For correspondence. (e-mail: forest@nwafu.edu.cn)
}

blished by Liu in the 1960s, is the most widely used and well-developed method. After decades of development and improvement, the prediction model based on probability integration successfully solved the problem of mining subsidence prediction in the plain mining areas of China, thanks in part to the area's simple geological condition. However, since the start of the 21 st century, the loess-covered mountainous terrain in Western China has become the main coal-producing area. Underground coal mining in these regions has not only led to generally well-understood mining subsidence deformation, but the combination of gravity and topographic relief has also caused the plastic slip deformation of the surface slope. This makes the mechanisms behind the land subsidence deformation more complex ${ }^{11-14}$. The thickness of surface soil layer in the western loess-covered areas is $60-300 \mathrm{~m}$, which represents $30-70 \%$ of the mining depth ${ }^{15}$. Because the physical and mechanical characteristics of the loess layer are different from those of the rock layer, the selfweight of the loess layer has a significant loading effect on bedrock subsidence and there is a complex coupling relationship between the loess layer and the bedrock in mining subsidence ${ }^{16-18}$. In summary, due to the influence of surface hillside slip and loess layer characteristics, the mining subsidence characteristics of loess-covered mountain areas in western China are considerably different from other mining areas in China, and the existing probability integration method for flat ground conditions and single rock medium is unsatisfactory for predicting the mining subsidence and deformation in western loesscovered mountain areas.

Using intensive computer modelling and ground truth validation $^{19-22}$, our team discovered the unique process and mechanisms of mining subsidence in loess-covered mountain areas of western China. In order to establish the prediction model of land subsidence and deformation, we treated mining subsidence in areas with thick loess covering the bedrock as the result of joint action of underground excavation and the loading imposed by the weight of the loess, and considered land subsidence deformation to be the result of non-uniform subsidence of the bedrock that passes up into the thick loess layers ${ }^{23}$. With these considerations, a mining subsidence prediction model for 
thick soil and bedrock double-layer medium was developed using the principles of stochastic medium theory. Moreover, during the process of mining subsidence of loess-mountains, the surface slopes will produce a significant lateral slip. In this study, we employed principles from soil mechanics to develop a model of slip on hillsides due to mining, and further combined this model with the conventional mining subsidence model. The combined model was then applied as a prediction model for mining subsidence and deformation in loess-covered mountain areas of western China.

\section{Double-medium model of mining subsidence in loess-covered mining areas}

\section{Effects of loading by loess layers on bedrock mining subsidence}

Figure $1 a$ illustrates the overlying bedrock and loess layer subsidence models driven by underground mining in mining areas with thick loess covers. Bedrock surface subsidence caused by underground coal mining is expressed by $W_{j}(x)$, and ground subsidence introduced by uneven subsidence of the bedrock surface is expressed as $W(x)$. As illustrated in Figure $1 b$, we also considered the impacts of loess layer on bedrock mining subsidence as the equivalent load of loess layer imposed on the bedrock surface. Many studies directly apply the soil weight of a static load $q_{0}$ on the bedrock control layer. This may be a reasonable assumption for loose sand or soft clay lay$\mathrm{ers}^{24}$. However, a structural layer of cohesive loess with a specific tensile and flexural strength, driven by the bedrock flexure process due to a loss of support, would actually form an 'arch' prior to structural collapse ${ }^{25}$. Meanwhile, part of the structure's weight would be transferred to the supporting rock on both sides, so that the bedrock surface available to withstand a static load is considerably less than the weight of the overlying soil layer.

To study the effects of loading imposed by loess layers on bedrock mining subsidence, we chose the Da Fo Si mine located in the Shaanxi province of China as a test area. Afterwards, we established a benchmark model based on the geological and mining conditions at this site
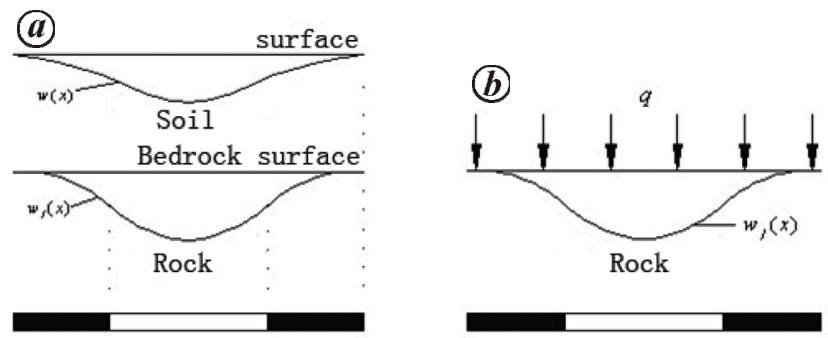

Figure 1. Mining subsidence model in loess-covered mining areas: $\boldsymbol{a}$, Land subsidence; $\boldsymbol{b}$, Bedrock subsidence activated by the equivalent load of the loess. and used the FLAC 3D model to simulate this effect. Meanwhile, the equivalent mining width $L_{w}$ was determined by the equivalent load of the loess layer, and the function relating the equivalent load imposed by the loess $q$, the weight of the loess layer $q_{0}$, and the actual mining width to depth ratio $\lambda$ was determined based on the numerical analysis result shown as

$$
\left\{\begin{array}{l}
q=0.5 \cdot q_{0} \quad \lambda \leq 0.35 \\
q=(0.33+0.48 \cdot \lambda) \cdot q_{0} \quad 0.35<\lambda \leq 1.4 \\
q=q_{0} \quad \lambda>1.4
\end{array}\right.
$$

In eqs (1), $q_{0}$ represents the weight of the loess layer in $\mathrm{MPa}$.

The function relating the equivalent mining width $L_{w}$, actual mining width $L_{z}$, the weight of the loess layer $q_{0}$ and the actual mining width to depth ratio $\lambda$ was determined based on the numerical analysis result shown as

$$
\left\{\begin{array}{l}
L_{w}=L_{z} \cdot\left(1+q_{0} / 10 \mathrm{MPa}\right) \quad \lambda \leq 0.35, \\
L_{w}=L_{z} \cdot\left(1+(0.67+0.95 \cdot \lambda) q_{0} / 10 \mathrm{MPa}\right) \quad 0.35<\lambda \leq 1.4, \\
L_{w}=L_{z} \quad \lambda>1.4
\end{array}\right.
$$

\section{Bedrock subsidence prediction}

Characteristics of land subsidence in mining areas mainly depend on mining methodology used as well as the bedrock destruction level. When underground mining cavity is small, the integrity of the bedrock structure is maintained, with only minor bending occurring at the bedrock surface. Increases in size of mining result in fracturing of the bedrock and formation of a subsidence basin on the surface of the bedrock. As the size of mine continuously increases, the bedrock surface will eventually reach the maximum possible subsidence under the regional geological and mining conditions, which means that it reaches a full subsidence state. According to the theory of mining subsidence, bedrock and ground surface subsidence is minor when it is under the state of bending subsidence. This case is rarely found in the loess mining area, and thus was not taken into account in our model. When bedrock is in the condition of fracture subsidence or full subsidence, its subsidence characteristics agree with the stochastic medium theory. The bedrock surface rupture mining subsidence calculation model was established in accordance with the existing probability integration method

$$
\left\{\begin{array}{l}
W_{j}(s, t)=\frac{1}{W_{j 0}} W_{j}^{0}(s) \cdot W_{j}^{0}(t), \\
W_{j}^{0}(s)=\frac{W_{j m}}{C_{s}} \cdot \int_{0}^{l_{w x}-2 d_{j}} \frac{1}{r_{j}} \cdot \exp \left(-\pi\left(s-d_{j}-\eta\right)^{2} / r_{j}^{2}\right) \mathrm{d} \eta \\
W_{j}^{0}(t)=\frac{W_{j m}}{C_{t}} \cdot \int_{0}^{l_{w y}-2 d_{j}} \frac{1}{r_{j}} \cdot \exp \left(-\pi\left(t-d_{j}-\zeta\right)^{2} / r_{j}^{2}\right) \mathrm{d} \zeta .
\end{array}\right.
$$




\section{RESEARCH ARTICLES}

In eq. (3), $s$ and $t$ represent the position coordinates of bedrock surface points on the main section along the strike and dip directions respectively, and the origin of the coordinates refers to the vertical projection of the lower-left corner of the working face on the bedrock surface. $W_{j}(s, t), W_{j}^{0}(s)$ and $W_{j}^{0}(t)$ represent the subsidence prediction functions for points, strike and dip of main section of bedrock surface respectively. $W_{j 0}$ and $W_{j m}$ are the maximum amount of bedrock subsidence under geological and mining conditions and fracture subsidence mode respectively. $l_{w x}$ and $l_{w y}$ are the equivalent exploitation width along strike and dip direction of the working face respectively. $d_{j}$ and $r_{j}$ are inflection point offset and major influence radius of the bedrock layer in probability integration method respectively. $\eta$ and $\zeta$ are integral variables along strike and dip direction of working face respectively. The maximum amount of bedrock subsidence $W_{j m}$ can be calculated according to the equation

$$
W_{j m}=m \cdot q_{j} \cdot \cos \alpha \cdot \sqrt{p_{j} \cdot \lambda_{x w}} \cdot \sqrt{p_{j} \cdot \lambda_{y w}} .
$$

In eq. (4), $q_{j}$ is regarded as the full mining subsidence coefficient of the bedrock without any loading. $m$ and $\alpha$ represent the seam thickness and dip angle respectively. $p_{j}$ is a parameter that describes the characteristics of the bedrock. For hard rock, medium hard rock, and soft rock, this quantity normally takes the values $0.7,0.8$ and 0.9 respectively. $\lambda_{x w}$ and $\lambda_{y w}$ are the equivalent width to depth ratios along strike and dip of main section (the main section refers to a profile which crosses the central point of the rectangle working face and parallels to its boundaries), which are the ratio of the equivalent exploitation width $L_{w}$ and exploitation depth $H$ under the equivalent load $q$ of the loess layer. When $p \cdot \lambda_{x w} \geq 1, p \cdot \lambda_{y w} \geq 1$, $p \cdot \lambda_{x w}=p \cdot \lambda_{y w}=1$.

$C_{s}$ and $C_{t}$ are the distribution coefficients of maximum amount of bedrock subsidence along strike and dip of main section, whose values fall within the range 0.0-1.0. In eq. (3), when $C_{s}=1, C_{t}=1, W_{j m}=W_{j 0}$, we get the full subsidence prediction function for the bedrock.

\section{Mining subsidence prediction model for ground}

\section{Ground subsidence prediction}

Surface mining subsidence deformation of the loess layer is the result of impacts of uneven bedrock land subsidence on the overlying soil. Using the subsidence functions $W_{j}^{0}(s)$ and $W_{j}^{0}(t)$ along the strike and dip of the main section, according to the principles of the probability integration method, the ground subsidence prediction functions of any point on striking and dipping main section are derived respectively as

$$
\left\{\begin{array}{l}
W(x, y)=\frac{1}{W_{m}} \cdot W^{0}(x) \cdot W^{0}(y), \\
W^{0}(x)=\int_{-a_{1}}^{a_{2}} q_{t} \cdot W_{j}^{0}(s) \cdot \frac{1}{r_{t}} \exp \left[-\pi(x-s)^{2} / r_{t}^{2}\right] \mathrm{d} s, \\
W^{0}(y)=\int_{-a_{3}}^{-a_{4}} q_{t} \cdot W_{j}^{0}(t) \cdot \frac{1}{r_{t}} \exp \left[-\pi(y-t)^{2} / r_{t}^{2}\right] \mathrm{d} t .
\end{array}\right.
$$

In eq. (5), $x$ and $y$ represent the position coordinates of any point in ground surface along the strike and dip direction respectively and the origin of coordinates is the vertical projection of the lower-left corner of the working face on the ground. $W(x, y), W^{0}(x)$ and $W^{0}(y)$ represent subsidence prediction functions of any point on striking and dipping main section in ground surface respectively. $W_{m}$ is the maximum amount of ground subsidence. $q_{t}$ is regarded as the subsidence coefficient of the loess layer. $a_{i}(i=1,2,3,4)$ signifies the coordinates of the left and right subsidence borders of the bedrock along the strike and dip of the main section. $r_{t}$ is the radius of major influence of loess layer in probability integration method. The maximum amount of ground subsidence $W_{m}$ can be calculated according to the equation

$$
W_{m}=q_{t} \cdot W_{j m} .
$$

Substituting eq. (3) into eq. (5), and setting the values of $C_{s}$ and $C_{t}$ to 1.0 , we then obtain the subsidence prediction functions of any point on striking and dipping main section of ground surface under the condition of full bedrock subsidence.

\section{Surface horizontal movement prediction}

The surface horizontal movement is considered to be the result of uneven subsidence in the soil spreading to the surface. The existing probability integration method was used to derive the formula describing the expected surface horizontal movement, assuming unit mining-induced rock (soil) deformation to maintain a constant volume. However, in mining areas with thick loess layers, the actual data and computer numerical analysis confirm that the range of horizontal movement on the surface is generally greater than the range of land subsidence, and the absolute values of horizontal and vertical deformations of the surface soil unit are different. They cause volume deformation of the soil unit mainly controlled by horizontal deformation. Through assuming the position coordinates of the soil unit as $(x, y)$, the horizontal movement function (eq. (7)) of the soil unit can be derived in terms of the derivation procedure of the horizontal movement formula established based on the probability integration method and the inter-relationships among horizontal 
deformation $E_{x}$, vertical deformation $E_{z}$ and volume deformation $E_{v}\left(E_{v}=E_{x}-E_{z} \neq 0\right)$

$$
\left\{\begin{array}{l}
U_{e}(x-s)=-\left[2 \pi B_{1} \cdot k_{0} \cdot(x-s) / r_{t}^{3}\right] \cdot \exp \left[-\pi(x-s)^{2} / r_{t}^{2}\right] \\
-\left[2 \pi B_{1 x}\left(1-k_{0}\right) \cdot(x-s) / r_{x}^{3}\right] \cdot \exp \left[-\pi(x-s)^{2} / r_{x}^{2}\right] \\
U_{e}(y-t)=-\left[2 \pi B_{3} \cdot k_{0} \cdot(y-t) / r_{t}^{3}\right] \cdot \exp \left[-\pi(y-t)^{2} / r_{t}^{2}\right] \\
-\left[2 \pi B_{3 y}\left(1-k_{0}\right) \cdot(y-t) / r_{y}^{3}\right] \cdot \exp \left[-\pi(y-t)^{2} / r_{y}^{2}\right]
\end{array}\right.
$$

In eq. (7), the first term on the right side refers to the horizontal displacement function of the soil unit derived based on the contemporary probability integration method. The second term refers to the function which is derived based on the contemporary probability integration method to describe the horizontal displacement of soil unit caused by the volume deformation $E_{v} . B_{1}$ and $B_{3}$ are horizontal movement scaling factors along the strike and dip of the main section respectively on the surface in the probability integration method. $B_{1 x}$ and $B_{3 y}$ are horizontal movement scaling factors caused by the volume deformation of soil unit along the strike and dip of the main section on the surface respectively. $r_{x}$ and $r_{y}$ represent the radii of influence of surface movement along the strike and dip of the main section respectively. $k_{0}$ is the horizontal deformation parameter, which is the ratio of the maximum vertical deformation and the maximum horizontal deformation of the surface unit. Other symbols have the same meaning as before. The prediction function of horizontal movement on the surface caused by uneven subsidence on the bedrock is derived as

$$
\left\{\begin{array}{l}
U^{0}(x)=\int_{-a_{1}}^{a_{2}} q_{t} \cdot W_{j}^{0}(s) \cdot U_{e}(x-s) \mathrm{d} t \\
U^{0}(y)=\int_{-a_{3}}^{a_{4}} q_{t} \cdot W_{j}^{0}(t) \cdot U_{\mathrm{e}}(y-t) \mathrm{d} t
\end{array}\right.
$$

Taking the first and second derivatives of the ground subsidence eq. (5) in $x$ and $y$ directions, the surface tilt $I^{0}(x)$ and $I^{0}(y)$ as well as curvature $K^{0}(x)$ and $K^{0}(y)$ of the striking and dipping main section can be obtained. By taking the first derivative of the eq. (8) describing the surface horizontal movement in $x$ and $y$ directions, the surface horizontal deformation $E^{0}(x)$ and $E^{0}(y)$ of the striking and dipping main section can be obtained. Due to the prescribed word limits, they are not listed.

\section{Methods for determining the prediction parameters}

(1) Bedrock subsidence factor: The full mining subsidence coefficient of the bedrock $q_{j}$ depends mainly on the compressive strength and thickness of the bedrock. Higher the bedrock strength, greater the degree of separation development and smaller the subsidence coefficient. When the thickness of the bedrock is greater, the subsidence factor also has a decreasing trend. For hard rock, medium hard rock, and soft rock, the subsidence coefficients are $0.50-0.65,0.65-0.80$ and $0.80-0.90$ respectively.

In the cases where actual data are available, $q_{j}$ can be determined according to the maximum amount of land subsidence $W_{0}$ that can be measured under the condition of full bedrock subsidence as

$$
q_{j}=\frac{W_{0}}{\left(1-\sqrt{H_{t}} / 100\right) \cdot m \cdot \cos \alpha} .
$$

The symbols in eq. (9) have the same meaning as before.

(2) Loess layer subsidence factor: The loess layer subsidence factor $q_{t}$ is mainly affected by the thickness of the loess layer and mining influenced coefficient, which can be calculated as

$$
q_{t}=n_{t 1} \cdot n_{t 3}\left(1-\sqrt{H_{t}} / 100\right)
$$

In eq. (10), $H_{t}$ is the thickness of the loess layer. $n_{t 1}$ and $n_{t 3}$ are the mining influenced coefficients of the loess layer respectively, whose values fall within the range 0.0-1.0. According to the result of computer simulation analysis, the relationship between $n_{t 1}\left(n_{t 3}\right)$ and the mining width to depth ratio $\lambda$ is shown in Figure 2 .

(3) Major influence radius: Major influence radius of bedrock subsidence $r_{j}$, soil subsidence $r_{t}$ and surface horizontal movement $r$, are all related to the thickness and characteristics of the bedrock or soil cover. They can be determined using the equations

$$
\left\{\begin{array}{l}
r_{j}=V_{j} \sqrt{H_{j}}, \\
r_{t}=V_{t} \sqrt{H_{t}}, \\
r=r_{j}+r_{t} .
\end{array}\right.
$$

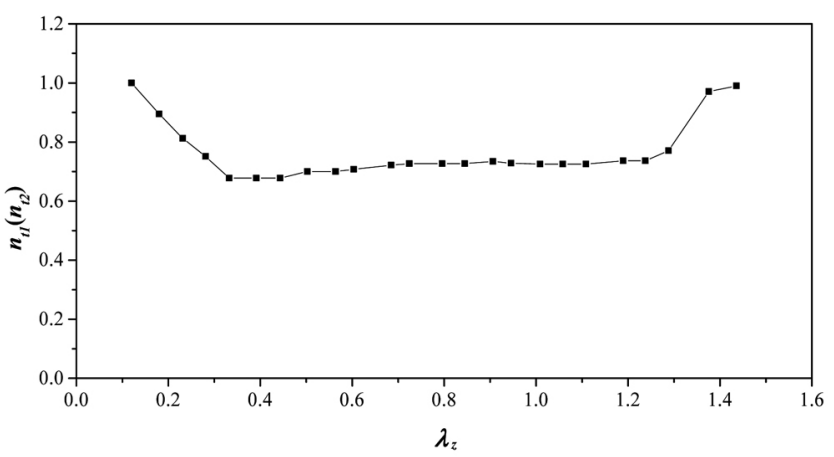

Figure 2. Relational curve between $n_{t 1}\left(n_{t 3}\right)$ and $\lambda_{z}$. 


\section{RESEARCH ARTICLES}

In eq. (11), $V_{j}$ and $V_{t}$ are the formation parameters. Their values increase with the strength of the rock or soil. When the bedrock is soft, medium or hard, the corresponding empirical value is $V_{j}=7.0-11.0$. The value for loess layer is $V_{t}=4.0-6.0$.

(4) Offset of bedrock inflection point: The bedrock inflection point offset $d_{j}$ is proportional to the thickness of the bedrock $H_{j}$, as indicated by

$$
d_{j}=f_{j} \cdot H_{j}
$$

In eq. (12), $f_{j}$ is a parameter describing the characteristics of the bedrock, which is integrated with the hardness of the bedrock. When the bedrock is soft, medium or hard, the corresponding empirical value of is $f_{j}=0.08-0.14$.

(5) Horizontal movement scaling factor: In the probability integration method, $B_{1}$ and $B_{3}$ are horizontal movement scaling factors caused by surface mining along the strike and dip of the main section respectively. $B_{1 x}$ and $B_{3 y}$ are horizontal movement scaling factors caused by volume deformation of soil unit on the surface along the strike and dip of the main section respectively. Horizontal movement scaling factors along the strike and dip of main section for the nearly flat seam can be considered equal and can be calculated as

$$
\left\{\begin{array}{l}
B_{1}=B_{3}=b \cdot\left(r_{j}^{2}+r_{t}^{2}\right)^{1 / 2}, \\
B_{1 x}=B_{3 y}=b \cdot\left(r_{j}^{2}+r^{2}\right)^{1 / 2} .
\end{array}\right.
$$

In eq. (13), $b$ is the surface horizontal movement factor, which is the ratio of the maximum horizontal movement and the maximum subsidence on the surface. It can be determined from measured data. In mining areas with thick loess layers, its empirically determined values fall within the range $b=0.25-0.40$.

(6) Horizontal deformation parameter: The variable $k_{0}$ represents the ratio of the maximum vertical deformation and the maximum horizontal deformation of the surface unit. However, according to the above definition, this quantity is not easy to determine, given that the main features of lateral development of land subsidence and horizontal movement are described based on major influence radius. $k_{0}$ can be calculated using the major influence radius of the bedrock, soil and surface movement by the equation

$$
k_{0}=\left(r_{j}^{2}+r_{t}^{2}\right)^{1 / 2} /\left(r_{j}^{2}+r^{2}\right)^{1 / 2} .
$$

Because, $r=r_{j}+r_{t}$, we know that $0 \leq k_{0} \leq 1$. For mining areas with thick loess layers, $k_{0}=0.5$.

(7) Length of the semi-basin of the bedrock subsidence: The length of the semi-basin of the bedrock subsidence refers to the distance between subsidence borders along the strike and dip of the main section to the central point of the basin. The horizontal projection of the distance between the border of subsidence basin and working face is less than $1.4 r_{j}$, so the sufficient accuracy of the parameter $a_{i}(i=1,2,3,4)$ in eq. (5) can be achieved via the equation

$$
a_{1}=1.4 r_{j}, a_{2}=l_{w x}+1.4 r_{j}, a_{3}=1.4 r_{j}, a_{4}=l_{w y}+1.4 r_{j} \text {. }
$$

The symbols in eq. (15) have the same meaning as before.

In conclusion, to predict mining-related subsidence in mining areas with thick loess layers, in addition to the geometric parameters describing the geological mining, the particular parameters that are required include the bedrock subsidence factor $q_{j}$, the parameters describing bedrock characteristics $V_{j}$ and $f_{j}$, the parameter describing soil characteristic $V_{t}$, the horizontal movement factor $b$, and the horizontal deformation characteristic parameter $k_{0}$. The remaining parameters are intermediate variables.

\section{Model for predicting slip deformation on loess hillsides due to mining}

\section{Prediction of additional horizontal movement and subsidence caused by hillside slippage}

In order to utilize the existing prediction modelled signed for flat ground, we considered the subsidence and deformation in loess-covered mountain slopes as combined result generated from the two separate subsidence processes: (1) mining subsidence in the flat ground; and (2) plastic slipping on loess hillsides. Ground truth validation and computer modelling analysis confirm that plastic slipping on loess hillsides is related to other factors such as mining subsidence, slope inclination, combination morphology of slope bodies and soil layer characteristics. By defining the slip characteristic parameters $p_{1}$ and $p_{3}$ along strike and dip direction of mining subsidence, and assuming that the surface inclination is $\theta-\mathrm{a}$ value which is positive when the slope is forward and negative when the slope is a reverse - we derived the function of the additional horizontal movement caused by slope sliding along the strike and dip of main section as

$$
\left\{\begin{array}{l}
\Delta U^{0}(x)=p_{1} \cdot \tan \theta \cdot W^{0}(x), \\
\Delta U^{0}(y)=p_{3} \cdot \tan \theta \cdot W^{0}(y) .
\end{array}\right.
$$

In eq. (16), the slip characteristic parameters $p_{1}$ and $p_{3}$ are related to slope morphology, as well as, physical and mechanical characteristics of the loess layer, and both of them can be determined by measured data. After projecting the additional horizontal movement caused by slope 
RESEARCH ARTICLES

Table 1. Geometric, characteristic and prediction parameters of Y905 face

\begin{tabular}{lcccccccc}
\hline Geometric parameters & $H_{j} / m$ & $H_{t} / m$ & $H_{0} / m$ & $m / m$ & $L_{x} / m$ & $L_{y} / m$ & $\alpha$ & $V_{j}$ \\
\hline Value & 72 & 110 & 182 & 1.94 & 300 & 102 & $9^{\circ}$ \\
Characteristic parameters & $V_{t}$ & $f_{j}$ & $k_{0}$ & $q_{j}$ & $b$ & $W_{m} / m m$ & $r_{j} / m$ & $r_{t} / m$ \\
Value & 5 & 0.11 & 0.5 & 0.75 & 0.30 & 1286 & 76.4 & 52.4 \\
Predicted parameters & $r_{x} / m$ & $d_{j} / m$ & $B_{1}$ & $B_{1 x}$ & $a_{3} / m$ & $a_{4} / m$ & $p_{3}$ & 480 \\
Value & 129 & 8 & 27.8 & 45 & 180 & 1.0 & 480 \\
\hline
\end{tabular}

Table 2. Surface slope angles along the striking main section of Y905 face

\begin{tabular}{lcccccccc}
\hline Position coordinates $/ m$ & $x \leq-40$ & -20 & 0 & 20 & 40 & 60 & 80 & 100 \\
\hline Slope angle $\theta$ & $0^{\circ}$ & $20^{\circ}$ & $20^{\circ}$ & $20^{\circ}$ & $15^{\circ}$ & $10^{\circ}$ & $5^{\circ}$ & 0 \\
Position coordinates $/ m$ & 120 & 140 & 160 & 180 & 200 & 220 & 240 & $x \geq 260$ \\
Slope angle $\theta$ & $-5^{\circ}$ & $-10^{\circ}$ & $-15^{\circ}$ & $-15^{\circ}$ & $-15^{\circ}$ & $-15^{\circ}$ & $-15^{\circ}$ & $0^{\circ}$ \\
\hline
\end{tabular}

sliding deviating from the vertical direction, we get the function for additional land subsidence caused by slope sliding along the strike and dip of the main section as

$$
\left\{\begin{array}{l}
\Delta W^{0}(x)=p_{1} \cdot \tan ^{2} \theta \cdot W^{0}(x), \\
\Delta W^{0}(y)=p_{3} \cdot \tan ^{2} \theta \cdot W^{0}(y) .
\end{array}\right.
$$

\section{Methods for determining the slip characteristic parameters}

The foregoing parameters in this model mainly include the slip characteristic parameters $p_{1}, p_{3}$ and the surface inclination $\theta$, whereas the remaining parameters are the same as in the flat ground conditions. The inclination $\theta$ belongs to the geometric parameter, which is determined by the slope of the ground along the main section line. The slip characteristic parameters $p_{1}$ and $p_{3}$ are determined by the horizontal movement of the point of maximum subsidence. Assuming that the maximum amount of surface mining subsidence is $W_{m}$ and that the horizontal displacement $\Delta U$ exists at the point of maximum subsidence, $\Delta U$ can be regarded as the additional horizontal movement caused by slope sliding. In addition, the slip characteristic parameters can be calculated as

$$
p=\Delta U /\left(\tan \theta \cdot W_{m}\right)
$$

The slip characteristic parameters can also be determined from the measured values of the subsidence and horizontal movement. For example, assuming the measured values of subsidence and horizontal movement at any point on the main section are $W(x)$ and $U(x)$ respectively, the corresponding prediction value of horizontal movement caused by mining for flat ground conditions is $U^{0}(x)$, and the corresponding ground slope is $\theta(x)$, then the slip characteristic parameter can also be calculated as

$$
p(x)=\left(U(x)-U^{0}(x)\right) /(\tan \theta(x) \cdot W(x)) .
$$

The slip characteristic parameters are determined by least square principle using the measured data. According to the analysis of the measured data, the value of this parameter is generally $0.5-1.5$.

\section{Application example}

This paper chose the Y905 face as a study site, which is associated with the main section of Yakou coal mine in the Tongchuan mining area, Shaanxi province, China. The established prediction model was utilized to calculate the surface mining subsidence deformation and the hillside slip deformation and further analyze the joint effect of these two deformations. Modelling results were then compared with the ground truth data to evaluate the accuracy of the prediction model.

\section{Prediction parameters}

The surface bedrock belongs to the medium-hard rock, and the geometric parameters of the mine, bedrock, and soil parameters and prediction parameters are shown in Table 1 . The value of slip characteristic parameter $p_{3}$ was set to 1.0 .

\section{Modelling result of land subsidence and horizontal movement under flat ground conditions}

The land subsidence and horizontal movement along the strike of the main section under flat ground conditions were calculated using eqs (5) and (8). The curves of land subsidence and horizontal movement are presented as curve A in Figures 3 and 4 respectively.

\section{Modelling result of surface slip deformation}

The inclination of surface, as shown in Table 2, was determined based on simple topographic maps and strike sections on the surface. 


\section{RESEARCH ARTICLES}

The land subsidence and horizontal movement along strike of main section caused by hillside slippage were calculated based on prediction position coordinates and corresponding slope angles calculated using eqs (16) and (17). The curves of land subsidence and horizontal movements caused by hillside slippage are shown as curve B in Figures 3 and 4 respectively.

By integrating the result for flat ground conditions with the result of slip deformation on hill slope surfaces, the curves, which are shown as curve $\mathrm{C}$ in Figures 3 and 4, were obtained to describe the combined subsidence and horizontal movement under mountainous conditions.

The curves of ground surface elevation along the strike of the main section are presented as curve $\mathrm{E}$ in Figures 3 and 4 respectively. A comparison of the characteristics of each curve indicates that the characteristics of sliding horizontal movement are related to the surface slope inclination and direction. When the mining direction is the same as the horizontal movement direction, the amount of horizontal movement increases; otherwise, it decreases. In the surrounding valley $(x=100 \mathrm{~m})$, the hillside slip is zero as a result of superimposition of two types of movement. There is a large horizontal displacement of the surface in the vicinity of the central point of the goaf with maximum subsidence.

The curves of land subsidence and horizontal movement along the strike of the main section, which are derived from the ground truth data acquired at the surface

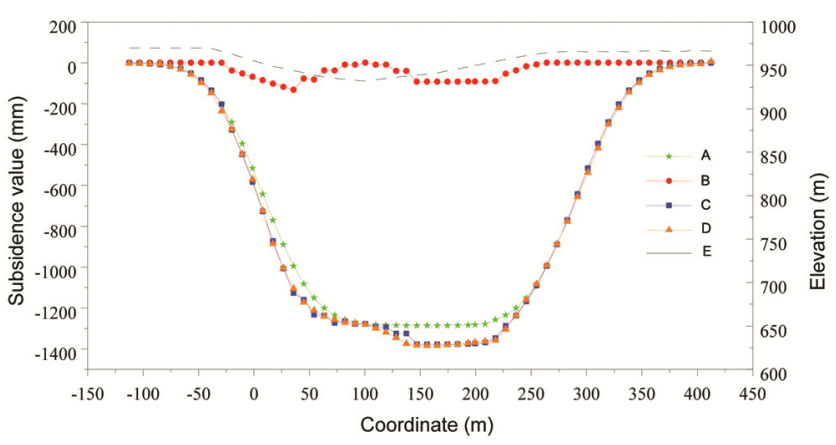

Figure 3. Superimposed land subsidence curves of the mountain.

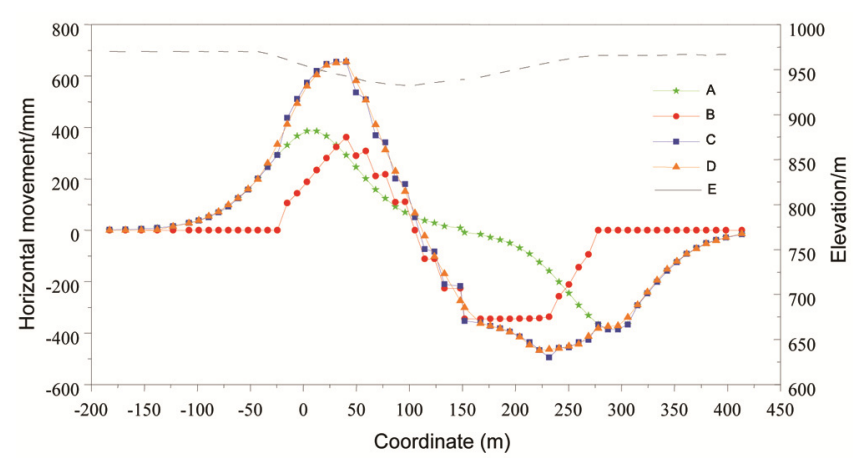

Figure 4. Superimposed horizontal movement curves of the mountain. movement observation station, are presented as curve D in Figures 3 and 4 respectively. Statistical analysis shows that modelling curve $\mathrm{C}$ closely matches the ground truth curve $\mathrm{D}$. The standard deviation of the subsidence prediction values of sample points is $8.8087 \mathrm{~mm}$, and the relative prediction error is $1.3 \%$. The standard deviation of the horizontal movement prediction values of sample points is $15.0139 \mathrm{~mm}$, and the relative prediction error was $4.5 \%$. Thus, the predicted results are demonstrated to be in agreement with the ground truth data.

\section{Conclusion}

(1) Land subsidence deformation in the loess-covered mountain areas of western China represents a combination of mining subsidence for flat ground conditions and hillside plastic slip deformation. The former can be decomposed into bedrock mining subsidence under equivalent loads of thick loess and deformation caused by uneven subsidence of the bedrock surface passing through overlying thick loess layers. In the loess-covered mountain slopes, the subsidence is related to factors such as mining subsidence, slope inclination, combination morphology of the slope, and soil layer characteristics.

(2) A relation exists between the equivalent load of loess layer $q$ and the mining width to depth ratio $\lambda$. Before the bedrock reaches its full subsidence state, the equivalent load of the loess $q$ is usually less than its own weight $q_{0}$. The double-medium model of mining subsidence in loess-covered mining areas is derived based on the stochastic medium theory by converting the equivalent load of loess layer into equivalent mining width.

(3) The application of the prediction model to the example given in this paper demonstrates that the land subsidence prediction model and the hillside slip deformation prediction model developed in our study are suitable for predicting mining subsidence and deformation in the loess-covered mountainous areas of western China or similar areas elsewhere. The new prediction model takes into account the differences between the soil and bedrock and the morphology characteristics of the surface, which represent the latest improvement in the process description and prediction accuracy of such models.

1. Zhang, J. X., Zhang, Q., Sun, Q., Gao, R., Germain, D. and Abro, S., Surface subsidence control theory and application to backfill coal mining technology. Environ. Earth Sci., 2015, 74, 14391448.

2. Howladar, M. F. and Hasan, K., A study on the development of subsidence due to the extraction of 1203 slice with its associated factors around Barapukuria underground coal mining industrial area, Dinajpur, Bangladesh. Environ. Earth Sci., 2014, 72, 36993713.

3. Álvarez-Fernández, M. I., González-Nicieza, C., Menéndez-Díaz, A. and Álvarez-Vigil, A. E., Generalization of the $n-k$ influence function to predict mining subsidence. Eng. Geol., 2005, 80, $1-36$. 
4. Zhu, X. J., Guo, G. L. and Fang, Q., Coupled discrete elementfinite difference method for analyzing subsidence control in fully mechanized solid backfilling mining. Environ. Earth Sci., 2016, 75, 683 .

5. Yang, D. J., Bian, Z. F. and Lei, S. G., Impact on soil physical qualities by the subsidence of coal mining: a case study in Western China. Environ. Earth Sci., 2016, 75, 652.

6. Wang, J. M., Wang, P., Qin, Q. and Wang, H. D., The effects of land subsidence and rehabilitation on soil hydraulic properties in a mining area in the Loess Plateau of China. Catena, 2017, 159, 51-59.

7. $\mathrm{Hu}, \mathrm{Z}$. Q. and Xiao, W., Optimization of concurrent mining and reclamation plans for single coal seam: a case study in northern Anhui, China. Environ. Earth Sci., 2013, 68, 1247-1254.

8. Wang, X. H., Hu, H. F. and Lian, X. G., Dynamic subsidence prediction method based on negative exponential method and Weibull time sequence function. Met. Mine, 2015, 44, 158-162 (In Chinese with English abstract).

9. Wu, X., Jiang, X. W., Chen, Y. F., Tian, H. and Xu, N. X., The influences of mining subsidence on the ecological environment and public infrastructure: a case study at the Haolaigou Iron Ore Mine in Baotou, China. Environ. Earth Sci., 2009, 59, 803810 .

10. Liu, B. C. and Dai, H. Y., Research development and origin of probability integral method. Coal Mining Technol., 2016, 21, 1-3 (In Chinese with English abstract).

11. Tang, F. Q., Research on mechanism of mountain landslide due to underground mining. J. Coal Sci. Eng., 2009, 15, 351-354.

12. Chang, Z. Q., Wang, J. Z., Chen, M., Ao, Z. R. and Yao, Q., A novel ground surface subsidence prediction model for sub-critical mining in the geological condition of a thick alluvium layer. Front. Earth Sci., 2015, 9, 330-341.

13. Tokgöz, Nuray, Case study of the Agacli landslide-gully complex during post-coal-mining reclamation and afforestation. Environ. Earth Sci., 2010, 59, 1559-1567.

14. Salmi, E. F., Nazem, M. and Karakus, M., Numerical analysis of a large landslide induced by coal mining subsidence. Eng. Geol., 2017, 217, 141-152.

15. Dai, H. Y., Lian, X. G., Liu, J. Y., Liu, Y. X., Zhou, Y. M., Deng, W. N. and Cai, Y. F., Model study of deformation induced by fully mechanized caving below a thick loess layer. Int. J. Rock Mech. Min., 2010, 47, 1027-1033.
16. Mulliken, J. S. and Karabalis, D. L., Discrete model for dynamic through-the-soil coupling of 3-D foundations and structures. Earthquake Eng. Struct. Dyn., 1998, 27, 687-710.

17. Guo, Q. B., Guo, G. L., Zha, J. F., Lv, X., Wang, J. T. and Chen, $\mathrm{T}$., Research on the surface movement in a mountain mining area: a case study of Sujiagou Mountain, China. Environ. Earth Sci., 2016, 75, 472 .

18. Zhou, D. W., Wu, K., Cheng, G. L. and Li, L., Mechanism of mining subsidence in coal mining area with thick alluvium soil in China. Arab. J. Geosci., 2015, 8, 1855-1867.

19. Tang, F. Q., Mining subsidence prediction model in western thick loess layer mining areas. J. China Coal Soc., 2011, 36, 74-78 (In Chinese with English abstract).

20. Zhang, X. S., Yu, H. C., Dong, J. Y., Liu, S. P., Huang, Z. Q., Wang, J. X. and Wong, H., A physical and numerical model-based research on the subsidence features of overlying strata caused by coal mining in Henan, China. Environ. Earth Sci., 2017, 76, 705.

21. Yang, W. F. and Xia, X. H., Prediction of mining subsidence under thin bedrocks and thick unconsolidated layers based on field measurement and artificial neural networks. Comput. Geosci., 2013, 52, 199-203.

22. Wang, J. M., Wang, P., Qin, Q. and Wang, H. D., The effects of land subsidence and rehabilitation on soil hydraulic properties in a mining area in the Loess Plateau of China. Catena, 2017, 159, 51-59.

23. Deck, O., Heib, M. A. and Homand, F., Taking the soil-structure interaction into account in assessing the loading of a structure in a mining subsidence area. Eng. Struct., 2003, 25, 435-448.

24. Lu, Q. Z. and Peng, J. B., Research on systematic frame and some problems of engineering geology of loess mass. J. Jilin Univ., 2006, 36, 404-409 (In Chinese with English abstract).

25. Wang, J. Z., Chang, Z. Q. and Chen, Y., Study on mining degree and patterns of ground subsidence in condition of mining under thick unconsolidated layers. J. China Coal Soc., 2003, 28, 230234 (In Chinese with English abstract).

ACKNOWLEDGEMENTS. This work was supported by the National Natural Science Foundation of China (No. 51674195, 41807063) and the Shaanxi Natural Science Foundation of China (No. 2016JM5048)

Received 12 January 2018; revised accepted 4 March 2019

doi: 10.18520/cs/v116/i12/2036-2043 